\title{
Aplicação de Robótica na Educação de Forma Gradual pa- ra o Estímulo do Pensamento Computacional
}

\author{
Débora Priscilla da Silva, Simone Shirley Sidnei, Ângelo Magno de Jesus, Carlos \\ Eduardo Paulino Silva \\ Instituto Federal de Minas Gerais, campus Ouro Branco \\ Rua Afonso Sardinha, 90 - Pioneiros - Ouro Branco / MG CEP: 36420-000 \\ deb sillva@hotmail.com, shirley_940@hotmail.com, \\ angelō.jesus@ifmg.edu.br, carlos.paulino@ifmg.edu.br
}

\begin{abstract}
This paper describes a teaching method of programing logics through robotics. This paper also reports the experience of applying this approach in a small town's public school. The main goal of this approach is to encourage the Computational Thinking of the students. As differential, the approach takes into account the progression on the Technologies Lego Mindstorms and Arduino. The method also takes into account the use of fewer units of robotics kits. This was made in order to suit the reality of these schools.
\end{abstract}

Resumo. Este artigo descreve uma metodologia de ensino de lógica de programação através da robótica e descreve um relato de experiência de sua aplicação em uma escola pública de um pequeno município. $O$ objetivo da abordagem é de estimular o Pensamento Computacional nos estudantes envolvidos nas atividades. Como diferencial, a metodologia proposta considera a progressão dos alunos entre as tecnologias Lego Mindstorms e Arduino. Além disso, a abordagem leva em consideração a utilização de poucas unidades de kits de robótica para se adequar a realidade das escolas públicas brasileiras.

\section{Introdução}

Do mercado, indústrias, medicina e até mesmo afazeres domésticos para sala de aula, a robótica vem ganhando grande espaço e interesse por parte de pesquisadores e professores de todo o mundo. Nos últimos tempos, a robótica se mostrou como uma ferramenta importante no processo de desenvolvimento cognitivo e de habilidades sociais dos alunos, desde a pré-escola ao ensino médio [ALIMISIS 2013]. Por isso, a robótica educacional pode ser um importante meio para motivar e envolver estudantes em atividades de programação e, consequentemente, trabalhar de forma lúdica o Pensamento Computacional. O estudante poderá ser motivado a criar um algoritmo pois terá a oportunidade de testar sua solução através das ações e interações de um robô.

Segundo Barr e Stephenson (2011) o Pensamento Computacional, no contexto 
do ensino básico, é uma abordagem de solução de problemas que pode ser automatizada, transferida e aplicada em diferentes matérias. Para os autores, os estudantes se tornam desenvolvedores das soluções. Barr e Stephenson (2011) também mostram como o Pensamento Computacional pode ser utilizado para trazer benefícios no aprendizado de diferentes disciplinas do ensino básico, como Matemática, Ciências, Estudos Sociais, Linguagem e Artes. Portanto, o estímulo do Pensamento Computacional pode trazer grandes benefícios para a educação básica brasileira, como um todo, uma vez que pode melhorar o desempenho dos alunos em várias disciplinas do currículo básico.

O presente trabalho se propõe a estimular o Pensamento Computacional de forma gradual em alunos do ensino fundamental, através de atividades de robótica realizadas em equipe. Para tal, a abordagem trabalha inicialmente conceitos teóricos de robótica e algoritmos, programação visual na tecnologia Lego EV3 e, finalmente, programação e desenvolvimento de projetos na plataforma Arduino. Isso em um contexto social de estudantes que pertencem a uma realidade local de uma escola pública de um pequeno município.

A metodologia proposta leva em consideração as limitações de disponibilidade de kits de robótica que geralmente são escassos ou inexistentes em escolas públicas brasileiras localizadas no interior. O trabalho também descreve a aplicação do método proposto em uma escola pública vinculada ao Programa Institucional de Bolsa de Iniciação a Docência (PIBID) do município de Ouro Branco. A experiência foi realizada com estudantes do sexto, sétimo e oitavo ano do ensino básico.

\section{Trabalhos Relacionados}

O ensino de conceitos de lógica de programação e algoritmos através da robótica tem sido explorado por pesquisadores e educadores de diferentes formas. No entanto, pode ser notado no trabalho de Neto et al. (2015) que ainda é necessário maiores esforços, por parte de pesquisadores, para que se tenha um maior número de trabalhos focados no tema Pensamento Computacional e Robótica Pedagógica. Ainda existem relativamente poucas publicações científicas envolvendo estes conceitos. Neste sentido, o uso da robótica no âmbito educacional é mostrado a seguir através de algumas iniciativas recentes com contextos aproximados ao presente trabalho.

Cambruzzi e de Souza (2015) desenvolvem, aplicam e avaliam Objetos de Aprendizagem baseados na Robótica Educativa com o objetivo de ensinar lógica de programação. Na abordagem dos autores, foram utilizados os kits de robôs da linha Lego Mindstorms em aulas de lógica de programação para alunos do curso técnico em Informática. Cambruzzi e de Souza (2015) concluem que a robótica educativa pode ser um elemento motivador e facilitador da compreensão dos conceitos trabalhados na disciplina de lógica de programação.

De Aguiar et al. (2015) descrevem a experiência pedagógica e o impacto da realização do projeto Lego nas Escolas. Em sua abordagem, foram convidadas turmas de oitava série de escolas públicas, que foram divididas em grupos, para realização de atividades de programação visual através dos kits de robótica Lego Mindstorms. Os autores observaram que, com a atividade, houve uma melhor adaptação por parte dos estudantes envolvidos às novas tecnologias. 
Raabe et al. (2015) relatam a experiência de uso de brinquedos de programar na Educação Infantil para resolução de problemas que envolvem conceitos matemáticos. Em sua abordagem os autores fazem um estudo de caso, com 15 crianças de 4 e 5 anos de idade, em que foram identificados indícios do potencial de desenvolvimento dos participantes.

Fiorio et al. (2014) apresentam uma experiência prática da inserção da robótica e seus benefícios como ferramenta educativa em escolas públicas estaduais de nível médio participantes do PIBID da UTFPR campus Francisco Beltrão. O trabalho envolveu a utilização de recursos tecnológicos, como os kits Lego Mindstorms, para auxiliá-los no processo de ensino-aprendizagem. Friedrich et al. (2012) propõe uma metodologia pedagógica para a ambientação e inserção de lógica de programação para crianças de sete a dez anos de idade. Os autores utilizam em sua abordagem as tecnologias Logo e Lego Mindstorms como meio auxiliar de ensino. Os resultados da experiência de aplicação da metodologia proposta por Friedrich et al. (2012) em uma turma de escola pública mostram que todas as atividades do plano de ensino foram concluídas com êxito pelas crianças. Segundo os autores, as crianças envolvidas se demonstraram receptivas e felizes por estarem participando do projeto.

Cardoso e Antonello (2015) relatam a experiência de utilização de programação visual e robótica, através de ferramentas de codificação por blocos e kits Arduino. O objetivo do trabalho foi ensinar conceitos de programação para alunos ingressantes no curso de Bacharelado de Sistemas da Informação. Os autores relatam que os resultados alcançados foram positivos. De França et al. (2014) apresentam as lições aprendidas com experiências de licenciandos em computação na disseminação do Pensamento Computacional na educação básica. Entre as experiências, os autores descrevem a realização de oficinas de robótica com o apoio do projeto ROBUCA: Inserção da Robótica Educativa no UCA e da Plataforma Robô Livre. Uma revisão sistemática da literatura a respeito da Robótica Pedagógica aplicada ao ensino de programação, através de laboratórios remotos, pode ser encontrada em Almeida e Netto (2015).

Segundo a análise do estado da arte realizada, identificamos que a robótica educacional tem sido utilizada para o ensino de lógica de programação de forma interdisciplinar, associada aos kits Lego Mindstorms (principalmente) ou ao Arduino. No entanto, as abordagens dos trabalhos analisados são focados exclusivamente em uma das tecnologias. A experiência realizada considerou o uso de ambas as plataformas Lego e Arduino. Além disso, a metodologia proposta neste artigo levou em consideração a limitação de unidades do kits de robótica devido a realidade das escolas públicas locais.

\subsection{O Uso dos Kits Lego Mindstorms e Arduino}

O Kit Lego Mindstorms é composto por vários tipos de sensores, que são responsáveis por coletar informações referentes ao meio externo, controlador, que é o elemento inteligente, motores (três no total), além de peças e componentes com funcionalidades especificas para a montagem do robô [Friedrich et al. 2012]. Já a plataforma Arduino tem ganhado grande fama pelo mundo. Isso ocorre devido a suas aplicações e ao fato de que pessoas, sendo especialistas ou não em programação e eletrônica, possam colocar em prática suas ideias de interação com objetos e ambientes. 
Nesta perspectiva, o Arduino pode ser considerado uma ótima possibilidade de aplicação em robótica pedagógica, pois além de ter baixo custo, também possui software livre [Pinto et al. 2012]. Contudo, a plataforma ainda não é das mais utilizadas. Alguns autores acreditam que para o uso de kits como o Arduino ainda é necessário certo conhecimento em eletrônica para o desenvolvimento de artefatos para robótica [Costa Junior; Guedes 2015]. Dessa forma, o Kit da Lego parece ser uma opção mais atrativa devido a sua maior facilidade de manuseio e construção de robôs.

\section{Método}

A abordagem proposta e aplicada neste trabalho tem como objetivo instigar o estudante na investigação e concretização dos conceitos de algoritmos e robótica. Portanto, o método é dividido em três etapas principais:

1. Introdução aos conceitos teóricos sobre Robótica e Programação.

2. Aplicação de atividades e desafios práticos de Programação Visual, com Lego Mindstorms, realizadas em grupo.

3. Realização de um projeto na tecnologia Arduino.

É importante observar que as etapas seguem uma sequência de evolução gradual do conhecimento dos alunos a respeito de lógica de programação e das tecnologias a serem utilizadas (Figura 1). No entanto, as etapas de trabalho também respeitam a autonomia dos estudantes para experimentarem e testarem diferentes soluções para os desafios propostos. Neste contexto, o Pensamento Computacional é trabalhado de forma que os alunos possam propor soluções lógicas que vão evoluindo a cada etapa.

A utilização do Lego, na etapa 2, permite introduzir os conceitos iniciais de programação e robótica, mais facilmente, preparando os alunos para trabalhar com plataformas mais complexas como a Arduino. A transição para tecnologia Arduino, na etapa 3, não se da de forma brusca, mas de forma gradual. Estando embasados em programação visual através do Lego, os alunos devem passar por aulas sobre programação em $\mathrm{C}$ do Arduino para iniciar a etapa 3. Após estas aulas, os estudantes, devidamente preparados, iniciarão as atividades do projeto da terceira etapa.

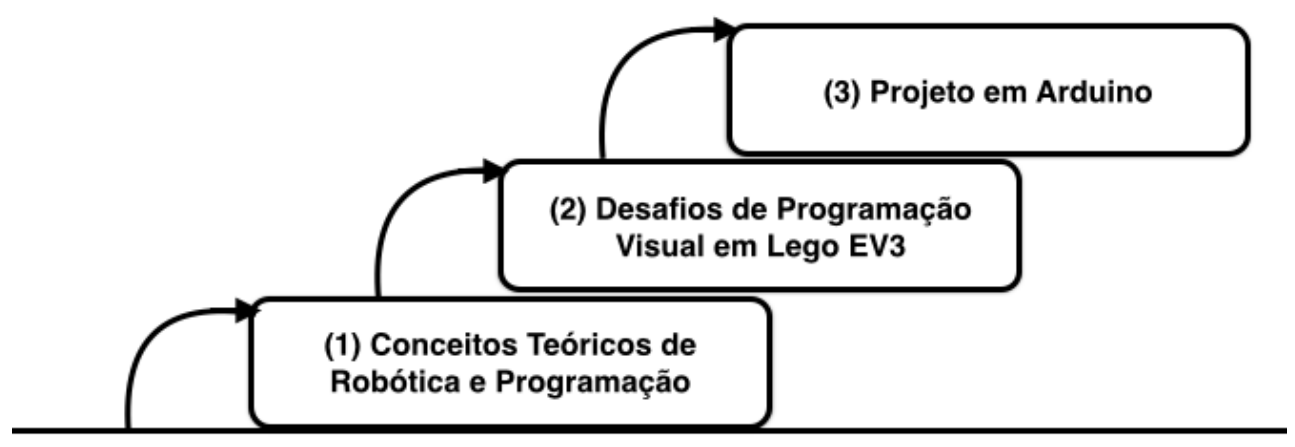

Figura 1: Etapas graduais do método proposto.

Recomenda-se a aplicação da abordagem proposta para estudantes a partir do 
sexto ano do ensino fundamental em turmas de aproximadamente 20 alunos. Recomenda-se também que as atividades sejam orientadas por dois tutores, que devem guiar todo o processo de aprendizado incentivando os discentes a participarem. O método também leva em consideração a disponibilidade de poucas unidades dos kits de robótica a serem utilizadas. Por isso, o formato da sala/laboratório para aplicação da atividade também é um fator a ser considerado. Para que os estudantes possam testar suas soluções, a sala deve ser organizada em semicírculo, no qual o centro, local onde o robô poderá se movimentar, será o espaço de testes. Esta organização de laboratório é ilustrada na Figura 2.

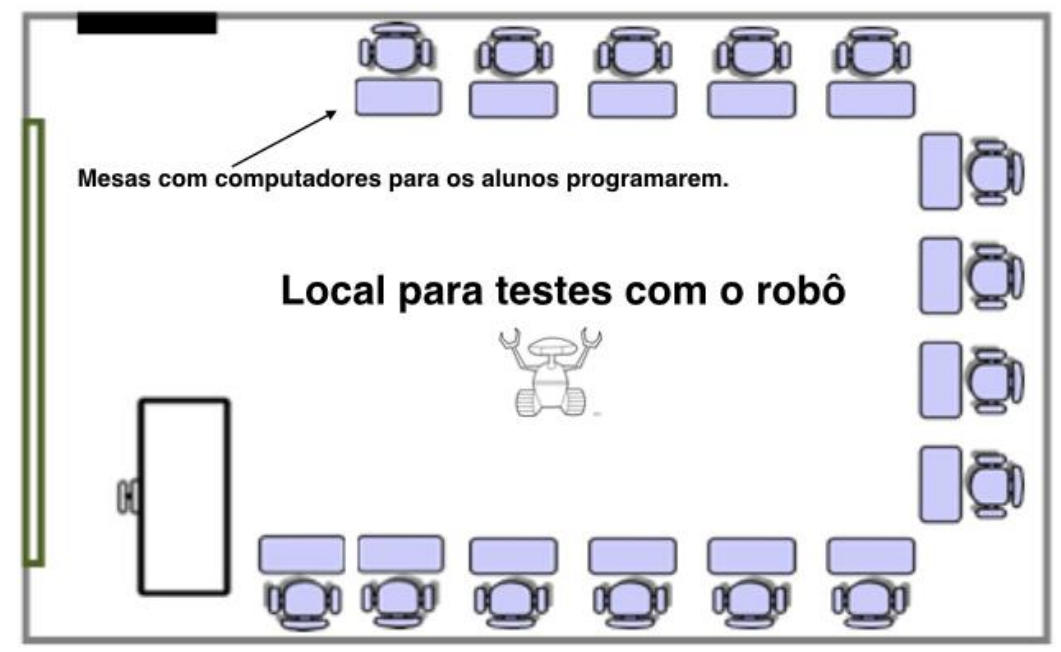

Figura 2: Formato da sala para aplicação das atividades práticas.

$\mathrm{Na}$ primeira etapa os tutores introduzem os fundamentos da robótica, são explicados conceitos teóricos a respeito de robôs e sua programação. Os tutores devem, não somente passar as informações, mas manter a participação ativa dos alunos, os questionando a respeito dos conceitos e, assim, incentivando os estudantes a expor seus conhecimentos sobre o tema. Os tutores podem discutir a temática robótica dentro do contexto da cultura popular incluindo filmes, desenhos animados e jogos eletrônicos. Isto pode instigar a curiosidade e aumentar o interesse das crianças. O robô também deve ser apresentado aos alunos no final desta etapa. Portanto, os tutores, devem manter um Robô Lego Mindstorms preparado para apresentação, assim como, para ser utilizado na etapa seguinte que envolve atividades de programação.

A escolha do modelo do Robô Lego a ser montado pelos tutores deve ser feita de acordo com os recursos (como sensores e atuadores) a serem utilizados nos exercícios práticos de programação. Além disso, é importante levar em consideração a criação de um robô com aparência que venha a ser "divertida" para o perfil da turma que se deseja trabalhar. Por exemplo, a montagem de um robô em forma de dinossauro pode agradar turmas mais jovens de estudantes.

A segunda etapa consiste na realização dos desafios de programação visual. Neste momento os alunos já devem ter passado pelos conceitos básicos de programação e devem, portanto iniciar a prática. A programação do robô, nesta etapa, pode ser feita através do software de programação visual padrão da própria fabricante Lego. Os tutores então devem apresentar uma série de desafios, que são atividades a 
serem realizadas pelo robô via programação. Os desafios devem ser realizados em uma série gradual onde os alunos vão subindo de nível a cada problema resolvido. Para incentivar a colaboração, os estudantes podem fazer a programação em duplas dividindo os computadores. Não é realizado nenhum tipo de competição entre os discentes, uma vez que, vencer o desafio em si já deve ser um fator motivador para realização da atividade. Os problemas, propostos pelos tutores, devem explorar os sensores e aturadores disponíveis no robô de forma que os discentes possam ver os resultados através das interações com o mesmo. Como a abordagem considera a limitação dos kits de Robótica, o robô fica sob responsabilidade dos tutores, que devem o disponibilizar quando um grupo precisar testar suas soluções. $\mathrm{O}$ tutor deve ficar responsável por fazer circular o robô entre os estudantes, desta forma todos o compartilham de maneira igualitária.

$\mathrm{Na}$ terceira e última etapa, os alunos devem desenvolver um projeto na plataforma Arduino. Trata-se de um desafio maior, uma vez que os estudantes devem entender conceitos mais avançados de programação e desenvolvimento de protótipos nesta tecnologia. Com o conhecimento adquirido nas etapas anteriores, os discentes passam por mais uma aula sobre alguns conceitos introdutórios sobre a plataforma Arduino. Esta tecnologia permite que os estudantes entendam como um robô pode ser montado e programado com variados componentes eletrônicos que podem ser de diferentes fabricantes ou re-aproveitados de lixo eletrônico. Recomenda-se o projeto de um semáforo automático, mas os tutores podem julgar e escolher outros projetos que se encaixem melhor no perfil e no grau de aprendizado dos alunos envolvidos na atividade.

\section{Experiência de Aplicação}

A experiência foi realizada com um grupo de 20 alunos composto por estudantes dos sexto, sétimo e oitavo anos do ensino básico. As atividades foram feitas no colégio municipal João XXIII, do Município de Ouro Branco, vinculada ao programa PIBID. Para realização das práticas de lógica programação, foi utilizado o próprio laboratório da escola que possuía organização e infra-estrutura mínima para acomodar os alunos e permitir o andamento dos trabalhos.

Na primeira etapa, os alunos se demonstraram bastante curiosos e interessados nos conceitos de robótica. Para preparação para a segunda etapa, o robô nomeado de Yuki, ilustrado na Figura 3, foi apresentada para os alunos. Os tutores escolheram o modelo Lego EV3STORM para montagem do Yuki por utilizar diversos sensores diferentes e, ainda, pela forma de interação e locomoção, adequados para a dinâmica das atividades propostas na Etapa 2. O modelo também foi escolhido por sua aparência ser bastante similar a muitos personagens de desenhos animados e filmes da cultura pop. 


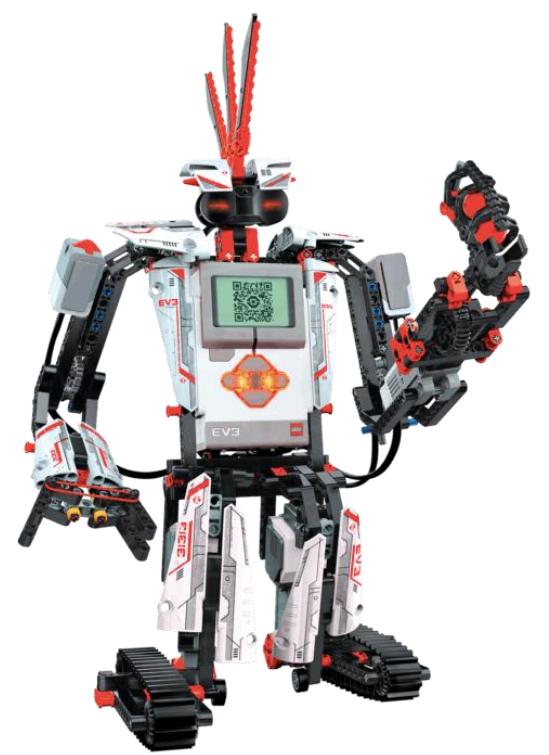

Figura 3: Modelo robô Lego Mindstorms utilizado na experiência.

$\mathrm{Na}$ segunda etapa, que consiste na realização dos desafios de programação, os alunos demonstraram cooperação no trabalho em equipe. Entre as atividades estavam: explorar os sensores do robô, simular um cachorrinho, fazer o robô "falar" nomes de cores, fazer o robô caminhar e desviar de obstáculos entre outras. A Tabela 1 mostra as principais atividades realizadas e a relação destas com os conceitos de programação explorados, incluindo a atividade da etapa 3 (Semáforo em Arduino) que será descrita a seguir. A Figura 4 ilustra os trabalhos realizados na escola.

Tabela 1. Relação entre as principais atividades e conceitos explorados na experiência prática

\begin{tabular}{|l|l|}
\hline \multicolumn{1}{|c|}{ ATIVIDADE } & \multicolumn{1}{|c|}{ CONCEITO EXPLORADO } \\
\hline $\begin{array}{l}\text { Andar, falar e mostrar } \\
\text { imagens no display }\end{array}$ & $\begin{array}{l}\text { Uso dos motores localizados nas pernas do robô, do display } \\
\text { e recursos de som. Aprendizado de conceitos estruturais } \\
\text { básicos da linguagem de programação como o iniciar e a } \\
\text { sequência de blocos de ação (que controla motores, sons e } \\
\text { imagens), bem como das peças contidas no robô. }\end{array}$ \\
\hline Desvio de obstáculos & $\begin{array}{l}\text { Utilização do sensor infravermelho para estimular o } \\
\text { aprendizado de estrutura condicional. }\end{array}$ \\
\hline Falar nome das cores & $\begin{array}{l}\text { Uso de estruturas de decisão simples e aninhada, repetição } \\
\text { e condição. Conceitos atrelados ao sensor de cor/luz. }\end{array}$ \\
\hline $\begin{array}{l}\text { Iniciar uma ação por } \\
\text { meio de "toque" }\end{array}$ & $\begin{array}{l}\text { Uso do sensor de toque e todos os outros conceitos } \\
\text { aprendidos nas outras atividades. Também usado como } \\
\text { reforço de conteúdo. }\end{array}$ \\
\hline Semáforo com Arduino & $\begin{array}{l}\text { Realizar atribuições as variáveis, bem como relacioná-las } \\
\text { por meio de operadores. Comparação de estrutura da } \\
\text { linguagem em blocos do Lego para do Arduino. }\end{array}$ \\
\hline
\end{tabular}




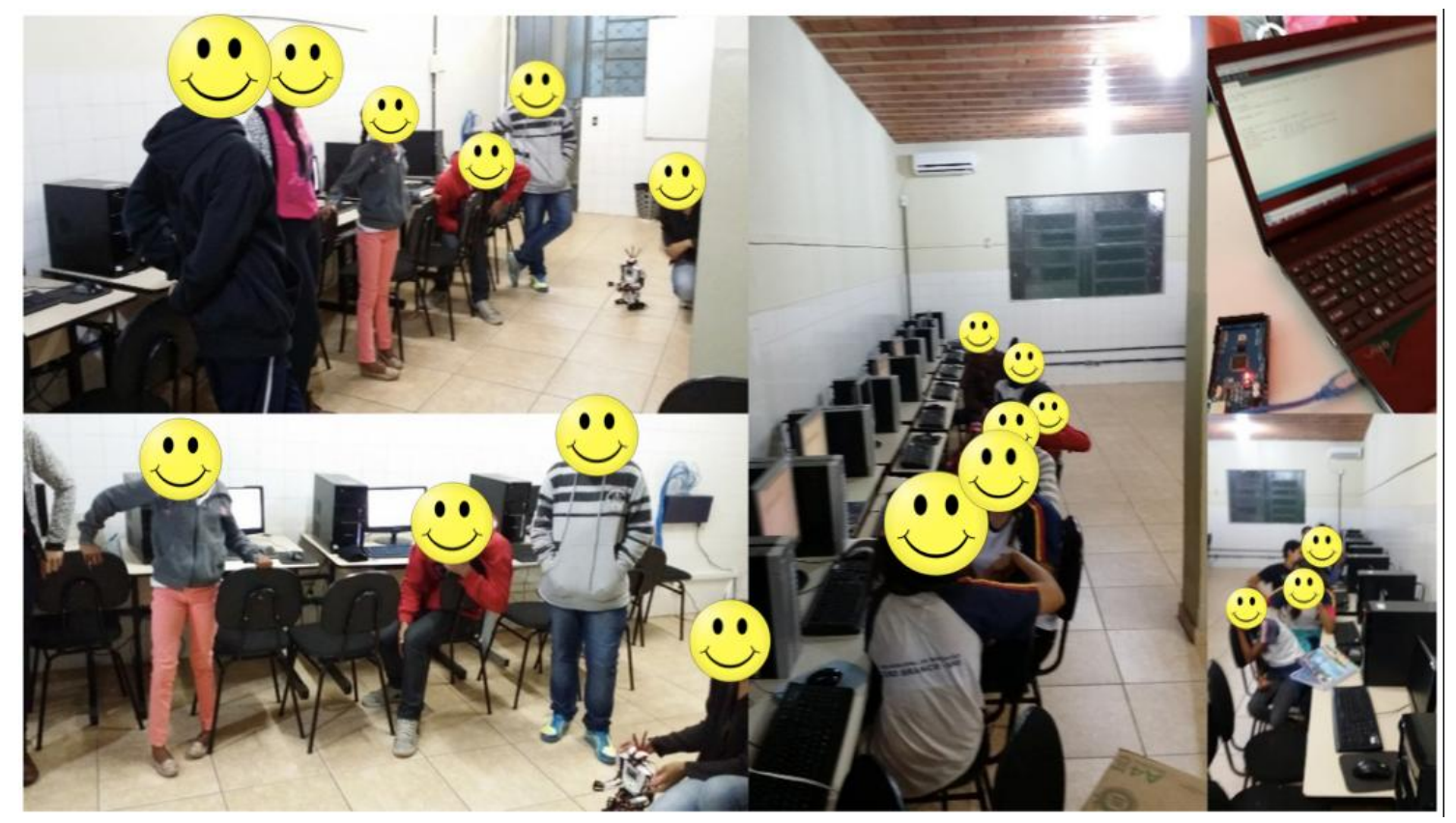

Figura 4: Alunos participando da segunda etapa da experiência.

Na terceira fase da oficina, consideremos que os alunos já estavam preparados para entender melhor o conteúdo básico da plataforma Arduino. Portanto, foram definidos conceitos como portas de entrada e saída, portas analógicas e digitais, projetos de circuitos microcontrolados, componentes do Arduino entre outros. Estes conceitos são estudados para que os tutores possam explicar a linguagem e forma usada para realizar a programação. A atividade inicial desta etapa consistia em fazer com que uma luz do tipo led piscasse em uma determinada frequência. Este exercício envolveu a ligação do circuito por meio do led, resistor e a placa; a verificação do funcionamento de diversos diodos emissores de luz; a utilização de portas digitais e temporização sequencial no Arduino. 3 nestes conceitos, os estudantes deveriam fazer o projeto de um mini semáforo, com tres leds de cores verde, amarela e vermelha, uma protoboard, tres resistors e fios jump. Desta forma, o aluno exerceu sua capacidade de investigação científica a partir do projeto desenvolvido.

\section{Resultados e Discussão}

Através da utilização da robótica, pôde-se observar que o projeto despertou nos alunos o interesse científico e os incentivou na solução de desafios que envolviam decomposição de problemas e realização de testes interativos através do robô, que são exercícios essenciais para estimular o Pensamento Computacional. Também foi possível observar a importância do trabalho em equipe na solução dos desafios propostos para os estudantes.

Quanto ao entendimento por parte dos alunos em relação aos conhecimentos apresentados e a aplicação dos mesmos na prática, consideramos os resultados 
satisfatórios. Por meio das atividades realizadas em laboratório foi possível observar o interesse e a motivação dos discentes que afirmaram que as ferramentas utilizadas foram essenciais para aprender conceitos de programação e robótica.

\section{Conclusões e Trabalhos Futuros}

Foi possível observar, durante a aplicação dos conceitos passados na oficina, que a robótica é um tema muito envolvente e efetivamente desperta o interesse dos alunos. Experiências têm indicado que o uso da Robótica Educacional nas escolas, para ensino de lógica de programação, possibilita que esses estudantes aprendam e aprimorem o Pensamento Computacional e desta forma tenham ganhos também no aprendizado de outras disciplinas.

O projeto é um grande sucesso entre os alunos. Portanto, como trabalhos futuros a proposta é sua continuação na escola, não apenas aplicando conceitos de programação e robótica, mas também a integração destes conceitos, principalmente no que diz respeito ao Pensamento Computacional, com os conteúdos de outras disciplinas. Desta forma, pode-se reforçar e ampliar o que já foi aprendido em sala de aula. Haverá também a aplicação de um questionário antes, durante e depois da oficina para avaliar quantitativamente seu aproveitamento e a evolução por parte dos alunos em outras disciplinas.

\section{Referências}

Alimisis, D. (2013), "Educational robotics: Open questions and new challenges". Themes in Science \& Technology Education, 6(1):63-71.

Almeida, T. O., J. Netto, J. F de M. (2015) "Robótica Pedagógica Aplicada ao Ensino de Programação: Uma Revisão Sistemática da Literatura", In: Anais do Simpósio Brasileiro de Informática na Educação. 26.

Barr, V. and Stephenson, C. (2011) "Bringing Computational Thinking to K-12: What is Involved and What is the Role of the Computer Science Education Community?", ACM Inroads, volume 2(1), 48- 54.

Cambruzzi, E., de Souza, R. M. (2015) "Robótica Educativa na aprendizagem de Lógica de Programação: Aplicação e análise", In: Anais do Workshop de Informática na Escola. 21.

Cardoso, R. e Antonello, S. L. (2015) "Interdisciplinaridade, programação visual e robótica educacional: relato de experiência sobre o ensino inicial de programação", In: Anais dos Workshops do Congresso Brasileiro de Informática na Educação. 4.

Costa Junior, Almir de Oliveira; GUEDES, Elloa B. (2015) "Uma Análise Comparativa de Kits para a Robótica Educacional", http://www.lbd.dcc.ufmg.br/colecoes/wei/2015/012.pdf. Acesso em: 13 maio 2015.

de Aguiar, Y. Q., Maciel, B. K., Mattos, S. D. G., Soares, L. B., de Oliveira, V. M. (2015) "Introdução à Robótica e Estímulo à Lógica de Programação no Ensino Básico Utilizando o Kit Educativo LEGO® Mindstorms", In: Anais dos Workshops do Congresso Brasileiro de Informática na Educação. 4.

de França, R. S., Ferreira, V. A. dos S., de Almeida, L. C. F., do Amaral, H. J. C. A 
V Congresso Brasileiro de Informática na Educação (CBIE 2016)

Anais dos Workshops do V Congresso Brasileiro de Informática na Educação (CBIE 2016)

(2014) "Disseminação do pensamento computacional na educação básica: lições aprendidas com experiências de licenciandos em computação", In: Anais do Congresso da Sociedade Brasileira de Computação. 34.

Fiorio, R., Esperandim, R. J., Silva, F. A., Varela, P. J., Leite, M. D., Reinaldo, F. A. F. (2014) "Uma experiência prática da inserção da robótica e seus benefícios como ferramenta educativa em escolas públicas", In: Simpósio Brasileiro de Informática na Educação, 25., Mato Grosso do Sul. Universidade Federal da Grande Dourados.

Friedrich, R. V., dos Santos, D. S., dos Santos K., R., Puntel, M. D., and Biasoli, D. (2012) "Proposta metodológica para a inserção ao ensino de logica de programação com logo e lego mindstorms.”, In Anais do Simpósio Brasileiro de Informática na Educação, 23.

Neto, R. P. B., Rocha, D. P., Santana, A. M., Souza, A. A. de S. (2015) "Robótica na Educação: Uma Revisão Sistemática dos Últimos 10 Anos", In: Anais do Simpósio Brasileiro de Informática na Educação. 26.

Pinto, M. d. C., da Fonseca Elia, M., e Sampaio, F. F. (2012). "Formação de professores em robótica educacional com hardware livre arduino no contexto um computador por aluno". In Anais do XVIII Workshop de Informática na Escola, volume 1.

Raabe, A., Rodrigues, A. J., Santana, A., Vieira, M. V., do Rosário, T., Carneiro, A. C. (2015) "Brinquedos de Programar na Educação Infantil: Um estudo de Caso". In: Anais do Workshop de Informática na Escola. 21. 